\title{
INVESTIGACIONES
}

\section{Diseño y evaluación de una guía de apoyo para fortalecer la tríada: alumno, profesor y familia}

\author{
Design and Evaluation of a Study Guide to Strengthen the Triad: Student, \\ Teacher and Family
}

\author{
Nielka Rojas González ${ }^{a}$, Dayana San Martín Toy \\ ${ }^{a}$ Universidad Católica del Norte. \\ Correo electrónico: nrojas03@ucn.cl

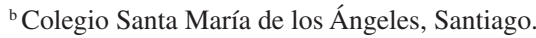 \\ Correo electrónico: dayana9403@live.cl
}

\begin{abstract}
RESUMEN
Este trabajo reporta los resultados del diseño y evaluación de una guía de apoyo para Tercer Año Básico en la asignatura de matemática, con el objetivo de fortalecer la tríada: alumno, profesor y familia. Empleamos una metodología cualitativa; como fuente de información realizamos Focus Group a profesores y alumnos, y entrevistas abiertas a profesores, las cuales otorgaron información para la construcción y evaluación del material diseñado. La guía de apoyo centrada en las fracciones fue evaluada positivamente por los profesores y familias. Destacando la propuesta por su diseño, el contexto de las actividades y la replicabilidad a otras asignaturas.
\end{abstract}

Palabras claves: familia, material educativo, fracciones, educación básica.

\section{ABSTRACT}

This work reports the results of the design and evaluation of a study guide for 3rd graders in the subject of mathematics. The aim of this work is to strengthen the triad: student, teacher, and family. A qualitative methodology was used for data collection. Focus groups were carried out with teachers and students, and open interviews were conducted with teachers. The information collected was used to construct and evaluate the material designed. The study guide designed to help students learn fractions was positively evaluated by teachers and families. The proposal was highlighted for its design, context of the activities, and its possible use with other subjects.

Keywords: family, instructional material, fractions, Primary Education. 


\section{INTRODUCCIÓN}

En este último tiempo se han disociando las funciones que cumplen las familias y las escuelas, a las primeras se les atribuyen exclusivamente una labor formativa valórica y la satisfacción de necesidades biologías, mientras que a las escuelas se les asocia con una labor de formación intelectual y académica (Arancibia, Herrera y Strasser, 1999; Romagnoli y Cortese, 2015). Dicho panorama genera un distanciamiento de las familias y los centros educativos, y a su vez la escuela siente como una intromisión la integración de las familias en el proceso de enseñanza aprendizaje.

Son múltiples las áreas de estudio que en estas últimas décadas han abordado la importancia de la participación de las familias en el proceso de enseñanza aprendizaje de sus hijos, concluyendo que no solo se ve beneficiado el estudiante, sino que también las instituciones educativas y todos los agentes sociales que intervienen en este proceso (Bolivar, 2006; Kaukab, 2016; Kotliarenco y Cortés, 2001; Razeto, 2016).

Es la escuela la que puede favorecer la cooperación con las familias, diseñando programas de trabajo enmarcados en sus proyectos educativos. Los programas no solo deben orientarse a actividades extra programáticas, sino destinarse a impulsar que la familia se implique en la formación de sus hijos y su desarrollo social.

El material de apoyo hacia la familia que elabora el MINEDUC se centra en abordar esencialmente temas transversales, por ejemplo el Manual para profesor jefe (UNICEF, 2007), Cartilla para padres, madres y apoderados/as (MINEDUC, 2011); entre otros. Siendo las prácticas más comunes generar un trato respetuoso hacia las familias, mejorar los canales de comunicación, accesibilidad del profesorado para atender a los apoderados, generar espacios y actividades variadas para distintos intereses de las familias. Esto muestra que existe una necesidad de incorporar a las familias en un ámbito más específico que impacte directamente en los aprendizajes disciplinares y en la integración de ellos. Como indica la UNICEF (2007) "la escuela necesita de los padres, requiere de su apoyo para el refuerzo de los contenidos trabajados en clases, para el cumplimiento de los aspectos formales que ésta exige y para asegurar que los niños reciban buenos tratos y afecto en sus hogares" (p.17).

En este sentido, el presente trabajo desarrolla una propuesta para fortalecer la triada alumno, profesor y familia proporcionado estrategias de apoyo al desarrollo integral del estudiante, a través de una guía para la familia, orientada a trabajar temas de la asignatura de matemática. La guía se focaliza en la asignatura de matemáticas, ya que a través de un diagnóstico inicial los estudiantes y las familias manifiestan interés por trabajar temas del área como división, multiplicación y fracciones.

La información obtenida del diagnóstico permite establecer criterios para el diseño del material de apoyo, abordándose el tema de las fracciones (significado parte-todo), para Tercer Año Básico en la asignatura de matemática.

Este trabajo concluye con la evaluación al material de apoyo que realizan las familias y docentes de un establecimiento de la región de Antofagasta.

\section{MARCO TEÓRICO}

A pesar de los diversos cambios que ha experimentado la conformación de las familias a nivel mundial, estas siguen siendo junto a las escuelas los principales agentes en la socialización 
de niños y jóvenes (Obiol, 2012). "Independiente del contexto y de su constitución, todas las familias cuentan con recursos para apoyar humanamente a sus hijos e hijas" (Gubbins, 2016: 152). Siendo tarea de las escuelas actuar para desarrollar esas capacidades, aspirando a la construcción de una sociedad más democrática, justa e inclusiva.

La familia es un agente fundamental en el aprendizaje de los niños y niñas; "es el primer ambiente social significativo que define y orienta pautas futuras en los esquemas de aprendizaje en general y en forma importante en los aprendizajes escolares" (Burrows, 2006:1).

De acuerdo a una serie de cambios sociales, económicos, culturales y tecnológicos que ha transformado la organización y funcionamiento de la familia, esta alude a variadas realidades: familias nucleares clásicas, miembros adoptivos, cohabitación irregular, hogares unipersonales, familias reconstituidas y familias monoparentales (Váldes y Váldes, 2005). De acuerdo a la heterogeneidad de realidades en este trabajo nos referimos a la familia, según definen Rodrigo y Palacios (2003) como aquellas personas que comparten un proyecto de existencia común, en el que se crean sentimientos de pertenencia a dicho grupo, además existe un compromiso personal entre las personas y se establecen relaciones de intimidad, reciprocidad y dependencia. De manera genérica, nos referimos a la familia como aquellas personas que orientan la labor educativa y que son los responsables frente a la escuela de la tutela de cada estudiante. Conformando la familia un pilar fundamental para el éxito académico de los estudiantes y de la comunidad en general.

González-Pienda y Núñez (2005) indican que como consecuencia de una relación homogénea de la escuela con la familia existen una serie de factores positivos, no solo para los estudiantes, sino también para la escuela y la familia. Entre los beneficios destacan el desarrollo de actitudes positivas de las familias, mayor satisfacción con los profesores, mejor entendimiento del funcionamiento del colegio y sus programas, mejor participación y responsabilidad en la escuela. En cuanto a la propia escuela, los profesores ven facilitada su tarea, alcanzando favorables resultados académicos y mayores beneficios escolares (Grant y Ray, 2013; Lucia y Breslau, 2006).

Considerando que el rol de las familias, además de cuidar, proteger y procurar el sustento de sus hijos, debe ser educarlos; es necesaria la participación para potenciar las capacidades e incidir en el éxito académicos de los estudiantes.

En el contexto meramente matemático los estudiantes de todos los niveles escolares necesitan dar sentido a los contenidos matemáticos que aprenden, para que puedan construir su propio significado de la matemática. Especialmente en los primeros niveles escolares, esto se logra cuando los alumnos exploran y trabajan manipulando material concreto. La formación de conceptos abstractos comienza a partir de las experiencias y acciones concretas con objetos, para más tarde avanzar progresivamente hacia un pensamiento simbólico-abstracto (González, 2010).

Considerando que en los primeros años de estudio es fundamental el acceso al conocimiento y el desarrollo de destrezas en los estudiantes, éstas pueden promoverse a través de situaciones problemáticas reales que generen la necesidad de utilizar herramientas matemáticas para su organización y solución. Por lo cual, es indispensable acercar a los estudiantes a imaginar situaciones a partir de realidades concretas o experiencias habituales, de modo que usen el sentido común, apliquen procedimientos de cálculo, estrategias de resolución que mejor sirvan para organizarlas (Heuvel-Panhuize, 2002). Lo anterior es un señalamiento que UNESCO (2004) realiza, enfatizando que los primeros educadores de 
los niños son las familias, por lo cual el espacio de aprendizaje por excelencia es el hogar, siendo la escuela un lugar para continuar y fortificar el conocimiento que la familia ha iniciado y sigue realizando. En este contexto es indispensable acercarnos a la construcción de conocimiento a partir del apoyo de las familias, pero ¿Cuáles son las técnicas que pueden utilizarse para estas tareas?

Por ejemplo, la UNICEF estudia las Escuelas Efectivas, entidades que no descuidan la relación familia-escuela, acogiendo a las familias de los estudiantes; además, las consideran como uno de los principales educadores. Existe un trato respetuoso hacia las familias, se informa en un lenguaje claro sobre lo que ocurre en el colegio, los directivos y profesores están disponibles para atender dudas y sugerencias a las familias, se estimula la presencia de las familias en espacios y actividades que realiza la comunidad (Corthorn y Pérez, 2005). Esta alianza de las Escuelas Efectiva, entre otras áreas, ha fortalecido la relación con las familias, buscando que estén presente en la sala de clase, brindando acompañamiento a los estudiantes para hacer las tareas en el hogar; buscando que los educandos alcancen metas formativas de calidad independientemente de su origen social (Raczynski y Muñoz, 2005).

El Ministerio de Educación chileno en los últimos años ha diseñado y distribuido en los distintos colegios municipales del país, una serie de guías para las familias, las cuales buscan ser una herramienta de apoyo pedagógico. Siendo el manual "Cuenta conmigo" uno de los documentos, que desde el año 2006 a la actualidad, ha impulsado la integración de las familias a la escuela (MINEDUC, 2016). Este manual presenta cuentos, leyendas, poemas, adivinanzas, juegos y textos informativos, que buscan que todos disfruten de la lectura y la relectura en familia.

Otra propuesta que presenta el Ministerio de Educación Chileno, en conjunto con la UNICEF, para trabajar con las familias es el "Manual para profesores jefes" (UNICEF/ MINEDUC, 2007). Este material apoya el trabajo del profesor en manejo de conflictos, motivación, problemáticas propias de la adolescencia, trabajo con las familias, (reuniones de apoderados), etc. Asimismo, la UNICEF (2009) propone el Manual para el Trabajo con Familias, para niños y niñas de 4 a 10 años, que se orienta a apoyar a las familias en hábitos de estudios propiamente tal.

En esta misma línea el MINEDUC ha desarrollado la propuesta "Reuniones con las familias", la que promueve explícitamente la generación de espacios democráticos y colaborativos de participación y encuentro entre escuelas y familias. Igualmente, la guía para apoyar a los padres y madres "Aprendiendo en familia" incentiva y orienta el rol educativo de las familias, buscando mejorar la comunicación y entregar orientaciones para disfrutar en familia (MINEDUC, 2013a). Presentando una serie de sugerencias sobre hábitos de estudios que deben incentivar en sus pupilos, en cómo enfrentar las tareas escolares, cómo lograr una buena comunicación en familia; además realizan actividades y generan recomendaciones para disfrutar en conjunto (rutina diaria, cocina en familia, la risa y el buen humor, jugando en familia, deporte en familia, entre otras).

Estos trabajos si bien están orientados para apoyar el aprendizaje escolar y fortalecer la relación familia-escuela, no son específicos en las orientaciones para que las familias apoyen a sus hijos en los estudios, en la realización de las tareas, en la adquisición de hábitos de estudios; es decir, en aspectos concretos de la formación académica.

La participación de las familias en la escuela no debe limitarse solo asistir a las reuniones escolares o reuniones extraescolares, deben participar en la organización habitual de sus hijos, ser partícipe de los aprendizajes que van adquiriendo, colaborar en 
propuestas o programas que sean favorable para el desarrollo de los estudiantes; por lo cual, la escuela debe ser un espacio para que la familia se siga formando, aprendiendo técnicas y estrategias para potenciar sus funciones (Corthorn y Pérez, 2005; De León, 2011; Villarroel y Sánchez, 2002).

En tal sentido, este estudio busca desarrollar y evaluar una propuesta metodológica que favorezca la tríada alumno, profesor y familia, focalizada en $3^{\circ}$ año básico en la asignatura de matemática. Concretamente se diseña y evalúa una guía de apoyo en que se aborda el tema de las fracciones (significado como parte-todo) con actividades prácticas para que las familias trabajen en sus hogares.

Las tareas matemáticas de la guía de apoyo se contextualizan en función al contexto personal definido por el Marco de PISA (2012); es decir, se presentan actividades que aluden a situaciones cotidianas para los alumnos, su familia y su grupo de iguales.

\section{METODOLOGÍA}

Empleamos metodología cualitativa para abordar la problemática planteada, ya que proporciona profundidad de información, riqueza interpretativa, contextualización del ambiente o entorno, detalles y experiencias únicas (Cohen, Manion y Morrison, 2011). Primero, efectuamos un diagnóstico en un establecimiento de la región de Antofagasta para identificar las necesidades de apoyo pedagógico en el área de matemática, para fortalecer la relación alumno, profesor y familia. El diagnóstico se realiza a partir de un Focus Group a los estudiantes de Tercer Año de escolaridad y apoderados del curso, también se realiza una entrevista abierta al profesor a cargo del curso. Luego, se diseña una guía de apoyo para trabajar un tema matemático con las familias y se examina el grado de satisfacción por parte de los apoderados, estudiantes, cuatro profesores de la asignatura de matemática y jefe de la Unidad Técnico Pedagógica (UTP).

El análisis de la información es descriptivo-interpretativo; es decir, se describen e interpretan las experiencias, necesidades, expresiones y emociones de los apoderados, estudiantes, profesor jefe y directivos respecto de una guía de apoyo para fortalecer el trabajo con las familias.

\subsection{SELECCIÓN DE LOS SUJETOS INFORMANTES}

Seleccionamos a un establecimiento subvencionado particular de la comuna de Antofagasta, ya que por disponibilidad de convenio con el centro se consiguió ingresar a sus aulas y a trabajar con las familias. El colegio es reconocido en la comuna por ofrecer educación de calidad, moderna, responsable y dinámica acorde al Proyecto Educativo (Decreto Nº12/2005 del Código del Derecho Canónico).

El Proyecto Educativo del establecimiento busca contribuir a la formación intelectual y valórica de los estudiantes y familias con interés en aspirar a niveles superiores de enseñanza. Asimismo, fomenta un ambiente de respeto mutuo y sana convivencia, responsable de transmitir y preservar el patrimonio cultural y valórico con un compromiso de servicio armónico, cordial y de excelencia académica.

Para fortalecer la relación con las familias, el establecimiento educativo realiza actividades como talleres de formación en el ámbito de convivencia escolar, drogadicción 
y alcoholismo, y actividades de recreación o esparcimiento como clases de baile, deporte o arte. Es decir, las actividades generadas por el establecimiento son recreativas; proporcionan placer y desarrollo de la personalidad, careciendo de actividades que fomenten integradamente el trabajo con estudiante y el apoderado.

Actualmente el centro educacional cuenta con una matrícula de 1.045 estudiantes, distribuidos en niveles de Pre Básica, Básica y Media. El grupo socioeconómico es de nivel medio, el nivel de escolaridad de los padres es de 11 años de estudios y la vulnerabilidad social de los estudiantes alcanza el $37,1 \%$.

Las características descritas del colegio muestran que existe ahínco por contribuir no sólo en la formación intelectual y valórica de los estudiantes, sino también en las familias; sin embargo, esto se queda en generar actividades extra programáticas que involucren a las familias. Siendo necesario actividades de participación de las familias en el proceso de enseñanza aprendizaje de sus hijos, como entregar indicaciones para que apoyen en los quehaceres de los estudiantes, brindar cursos de nivelación de estudios para padres y apoderados con estudios incompletos, etc. (UNICEF, 2005).

Concretamos la experiencia docente al nivel escolar de Tercer Año Básico del colegio descrito. Este nivel es considerado como etapa altamente sensible en la Enseñanza Básica, ya que se genera la transición al siguiente nivel escolar, por lo que los estudiantes necesitan generar un apoyo familiar sólido que les permita afrontar con mayor facilidad al cambio de ciclo escolar. También es una etapa en el cual los niños y niñas se encuentran en pleno descubrimiento de nuevas experiencias; comienzan a construir las bases de formación del aprendizaje, las cuales se irán desarrollando cada vez más, de acuerdo a las necesidades que el medio va exigiendo. En este periodo los estudiantes poseen un conocimiento intuitivo de nociones matemáticas sencillas, tienen un conocimiento inicial sobre números, siendo la base para el aprendizaje de la matemática formal y cada vez más compleja.

Otro aspecto fundamental en este periodo es la asimilación del aprendizaje; es decir, los estudiantes deben relacionar lo que aprenden en la escuela a otros contextos, siendo las familias un apoyo fundamental para evitar que el proceso de enseñanza aprendizaje de la matemática tenga un quiebre de la escuela a la casa. Así, las familias y las escuelas deben trabajar en conjunto y tener una labor formativa cohesionada e integrada.

Para este estudio también consideramos a la profesora jefe del curso y a la totalidad de apoderados o padres del curso de Tercer Año Básico para efectuar el diagnóstico.

\subsection{DIAGNÓSTICO}

El diagnóstico se realiza en el colegio con el objetivo de identificar las necesidades de apoyo pedagógico en el área de matemática, de modo de fortalecer la relación alumno, profesor y familia. Para ello, se recoge información a partir de un Focus Group a los estudiantes de Tercer Año de escolaridad y apoderados del curso, también se realiza una entrevista abierta al profesor a cargo del curso.

Consideramos el Focus Group, ya que genera una interacción discursiva y permite contrastar opiniones de los participantes de los grupos, siendo una forma para aprender de ellos a partir de la interacción discursiva del grupo (Gurdían, 2007). En el caso de los apoderados, permite recabar información con respecto a las fortalezas y debilidades que tienen sobre los contenidos matemáticos, competencias y habilidades transversales necesarias para apoyar a sus hijos en su formación académica. En el caso de los estudiantes, 
permite identificar las dificultades que presentan en la asignatura, grado de motivación, hábitos de estudios, apreciaciones sobre el rendimiento académico de la asignatura de matemática.

Las opiniones emanadas de las reuniones con los padres y los estudiantes se transcriben en turnos de palabras de cada integrante, luego se categorizan las opiniones más significativas y se interpretan para conocer las necesidades de apoyo pedagógico en el área de matemática (Padua, 2000).

Otro instrumento empleado en la etapa de diagnóstico es la entrevista abierta al profesor de la asignatura de matemática, con el objetivo de identificar debilidades en la adquisición de habilidades matemáticas en que los estudiantes del curso necesitan apoyo; es decir, conocer características generales y específicas, presencia de necesidades educativas especiales, estilos de aprendizaje, características socioculturales, entre otras.

La información obtenida de los grupos focales y entrevistas semiestructuradas son la base para el diseño de la guía de apoyo. En lo que sigue se presenta el análisis de cada Focus Group y entrevista.

\subsubsection{Análisis del Focus Group de los estudiantes}

Para recoger antecedentes del curso, se realiza un Focus Group con la totalidad de estudiantes que lo conforman (32 alumnos), los datos recogidos sirven para orientar la guía de apoyo a las familias. La interacción se producen en torno a dos temas: acompañamiento de las familias en el proceso de enseñanza y sobre los contenidos matemáticos de mayor complejidad.

Respecto al acompañamiento que reciben los estudiantes en el proceso de enseñanza, manifestaron variadas opiniones. Principalmente se destaca el apoyo de la mamá, abuela o de algún hermano para realizar las tareas, otros estudiantes trabajan de forma individual o simplemente no hacen los deberes. Por ejemplo, indican “[...] Mi mamá a veces me ayuda a hacer las tareas, cuando llega temprano de su trabajo, o cuando no tiene que ir a trabajar, pero a veces no entiende mis tareas"; existiendo apoyo de las familias, aunque en ocasiones no se sabe cómo enfrentar la enseñanza de algunos tópicos.

Respecto a ¿qué es lo más difícil de aprender en matemática?, los estudiantes expresaron que aprender la hora, las fracciones y las multiplicaciones. Enfatizando que las fracciones es un tema que "no entienden" y que es "difícil".

\subsubsection{Análisis del Focus Group de los padres y apoderados}

La discusión se realiza según las necesidades y/o debilidades que tienen las familias en el proceso de enseñanza aprendizaje y sobre los contenidos matemáticos.

Con respecto a que si las familias apoyan el proceso de enseñanza-aprendizaje de sus hijos, indican que es complejo el acompañamiento diario, ya que los alumnos llegan cansados al hogar. Estableciendo que el acompañamiento generalmente se produce cuando tienen evaluaciones o al final del semestre.

Cuando se consulta a los apoderados ¿cuáles son las debilidades que tienen para apoyar el proceso de aprendizaje de sus hijos?, indican que son mayormente en la asignatura de matemáticas. Precisando que "hoy se enseña diferente a sumar, restar y dividir", y a veces con los métodos que los padres o apoderados conocen, tienen a confundir a sus hijos. 
Con respecto a los contenidos matemáticos en los cuáles les gustaría recibir apoyo, mencionan las divisiones, como enseñar la hora y en su gran mayoría señalan que el tema matemático más complejo son las fracciones. Contenido que se continúa trabajando en el siguiente año académico. Concretamente indican "a mí también me gustaría que me enseñaran como ayudar a mi hija a estudiar las fracciones, yo he buscado ejercicios en internet, pero es muy aburrido; que después de estar aquí en el colegio todo el día después vaya a la casa a hacer más ejercicios".

\subsubsection{Análisis de la entrevista con la profesora jefe de la asignatura}

Realizamos una entrevista de preguntas abiertas a la profesora de la asignatura de matemáticas, para recabar información de diversos aspectos académicos y no académicos del curso. La profesora tiene seis años de experiencia como profesora general en Educación Básica, tiene un Pos Título en Teología. Ella manifiesta que le gustaría especializarse en nuevas metodologías para enseñar matemáticas, indicando "si no me actualizo luego, voy a quedar atrasada con lo que necesitan los niños", "he tenido que volver a estudiar varios contenidos de matemáticas y he pedido ayuda a otras colegas que ya han trabajado en Tercer Año Básico".

La profesora coincide con los alumnos, sobre los contenidos que los estudiantes presentan mayores dificultades, indicando "lo que es más difícil para ellos, son las fracciones, las multiplicaciones y la hora".

Con respecto a la relación y canales de comunicación que tiene la profesora con las familias de los estudiantes, indica que ellos son responsables, pero que en general ella no brinda tareas para el hogar, ya que los alumnos no las hacen, prefiriendo que trabajen en la clase.

\subsection{DISEÑO DE LA GUÍA DE APOYO}

A partir de las consideraciones resultantes del diagnóstico y de acuerdo a las orientaciones curriculares para el nivel de Tercer Año Básico y manuales preexistentes para fortalecer la triada alumno, profesor y familia se elabora la guía de apoyo para las familias, en el área de matemática, atendiendo al contenido de las fracciones

La propuesta se orienta a lograr un apoyo específico en la asignatura de matemática de $3^{\circ}$ Año Básico, se utiliza como fuente principal las bases y programas de estudio vigentes, las cuales proporcionan las orientaciones pedagógicas para la guía de apoyo a las familias.

En cuanto al contexto meramente matemático la implementación de esta propuesta tiene por objetivo el fortalecimiento de habilidades matemáticas sobre el tema de fracciones. Considerando que el propósito general que tiene la asignatura de matemáticas es enriquecer la comprensión de la realidad, facilitar la selección de estrategias para resolver problemas y contribuir al desarrollo del pensamiento crítico y autónomo de todos los estudiantes.

El material fue diseñado, siguiendo el objetivo de aprendizaje $\mathrm{N}^{\circ} 11$, del eje de Números y Operaciones para Tercer Año Básico: "Demostrar que comprenden las fracciones de uso común: $\frac{1}{4}, \frac{1}{3}, \frac{1}{2}, \frac{2}{3}, \mathrm{y} \frac{3}{4}$. Explicando que las fracciones representan la parte de un todo, de manera concreta, pictórica, simbólica, de forma manual y/o con software educativo. Describiendo situaciones en las cuales pueden usar fracciones. Comparando fracciones de un mismo todo, de igual denominador." (MINEDUC, 2013b). Por lo cual, la guía se centra 
en trabajar el significado de fracción como parte-todo, reforzando el significado de fracción que deben comprender en Tercer año Básico.

Los elementos estructurante de la guía de apoyo familiar son los siguientes: orientaciones generales para las familias y motivacionales; desarrollo del tema matemático y ejemplificación; ejercicios; actividad práctica para trabajar en familia; páginas web para apoyar la enseñanza; y, problemas matemáticos en contextos familiares. En lo que sigue se presenta la guía de apoyo elaborada. Su aprendizaje involucra capacidades para visualizar, representar y resolver problemas. La matemática está siempre presente en la vida cotidiana, explícita e implícitamente y juega un papel fundamental a la hora de tomar decisiones, estando la guía contextualizada para trabajar en familia en contextos personales. A continuación presentamos la guía de apoyo familiar elaborada.

\section{GUÍA DE APOYO FAMILIAR \\ "Educar tarea de dos". Educación cosa de dos, Familia - Escuela ¿Sabía usted qué?}

Si mejoramos la comunicación de la familia, la escuela y el profesor, se puede comprender de mejor manera el contexto de cada estudiante, favoreciendo la selección de estrategias educativas adecuadas para el proceso de aprendizaje de cada alumno.

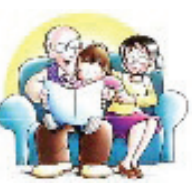

Aquellos estudiantes que no sienten el apoyo de sus familias en su proceso de enseñanza aprendizaje, triplican los riegos de sufrir enfermedades relacionadas con el estrés. Existe también una directa relación entre el apoyo de las familias y el tipo de comportamiento que tienen los estudiantes, el rendimiento escolar y el sentimiento de seguridad (Coleman y Hendry, 2003).

A continuación los invitamos a realizar estos pequeños cambios:

No pregunte si le fue bien o mal, sino pregunte: ¿Qué hiciste hoy en el colegio?

Pregunte al estudiante: ¿Qué aprendió hoy?

Pregunte al estudiante: ¿Qué fue lo que lo hizo más feliz? 


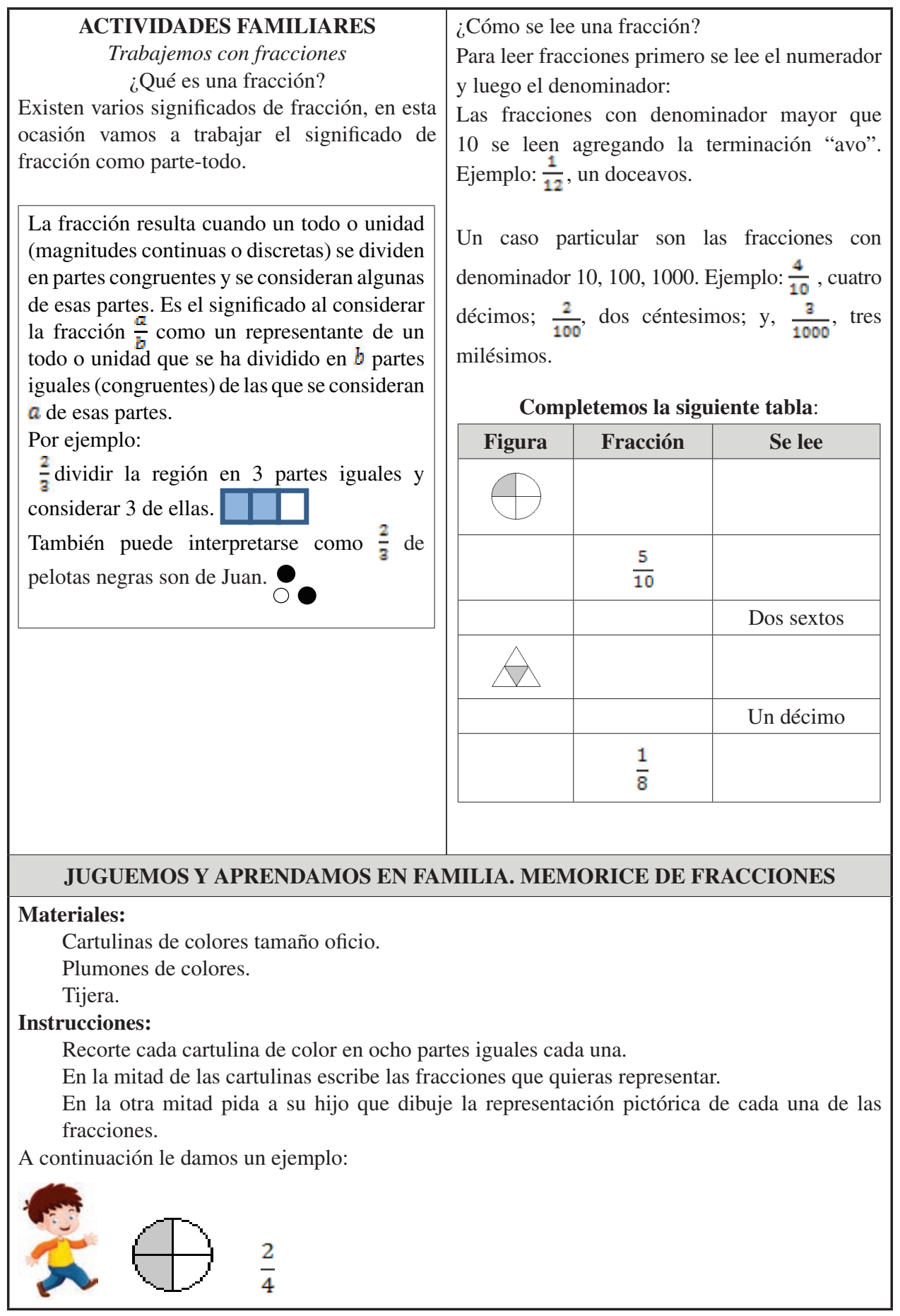




\section{Instrucciones del juego:}

- Cuando todas las fichas estén completas reúnanse en familia. ¡Y a jugar!

- Coloque todas las fichas con las fracciones y sus representaciones hacia abajo.

- Por turnos cada jugador debe dar vuelta dos fichas, con el objetivo de ir armando los pares correspondientes.

- Si las fichas no coinciden, deben dejarla en el mismo lugar que estaban anteriormente.

- Gana el jugador que logre armar más pares de fracciones con sus respectivas representaciones.

Sabemos que los estudiantes pasan gran parte de su tiempo en Internet.

A continuación le recomendamos una serie de páginas de Internet, en que pueden aprender sobre las fracciones y divertirse viendo videos, realizando actividades, ejercitando, etc.

https://youtu.be/wtarlG2TM_w

https://youtu.be/ODQpnt06M0M

http://www.curriculumenlineamineduc.cl/605/w3-propertyvalue-57984.html

http://www.curriculumenlineamineduc.cl/605/articles-32570_recurso_html.html

http://www.curriculumenlineamineduc.cl/605/articles-32572_recurso_html.html

\section{COMPROBANDO LO APRENDIDO EN FAMILIA}

Pida a su hijo o pupilo que divida su pan en 4 partes iguales, luego dígale que le indique la fracción que representa una parte, dos partes, tres partes y cuatro partes.

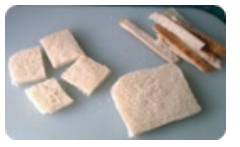

Sirva cuatro vasos de agua $(250 \mathrm{cc})$ para los integrante de la familia, pregunte a su hijo o pupilo, ¿Qué fracción representa la cantidad de agua de un vaso? ¿Y de tres vasos? (respecto del litro de agua).

Busque un calendario, elija el mes que desee y pregun al estudiante lo siguiente:

(Considerando la totalidad de días del mes como el entero).

¿Qué fracción del mes representan los días lunes?

¿Qué fracción del mes representan los días feriados?

¿Qué fracción del mes representan los días de clases?

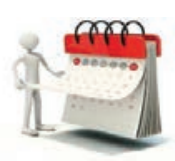

Repasaremos las fracciones a la hora del té, si el entero queda representado por todos los platos que estan en la mesa (platos iguales). Pregunte al estudiante lo siguinete:

¿Qué parte de la fracción representa el plato del estudiante?

¿Qué fracción representaría la totalidad de platos de la mesa?

Si quitara dos platos, ¿Qué fracción representaría la totalidad de platos que quedaron? 
La guía fue distribuida a los apoderados del Tercer Año Básico del colegio descrito, con el objetivo que las familias aplicaran los ejercicios y actividades propuestas con sus hijos o pupilos. El material fue enviado a las familias a través de los estudiantes, junto a una carta de presentación y una encuesta para saber su grado de satisfacción respecto al material. La totalidad de las familias del curso (32), recibió su encuesta de satisfacción, de las cuales 26 de ellas enviaron sus apreciaciones.

Además, la guía de apoyo para la familia y la encuesta de satisfacción aplicada fue validada por tres docentes expertos en el área de matemáticas; ellos indicaron que los contenidos matemáticos están claramente definidos y explicados, realzándose que "los ejercicios de fracciones presentes en la guía, si bien trabajan la fracción como parte-todo, que es lo idóneo para Tercer Año Básico, también articula la fracción como medición que se estudiará en los cursos superiores, como es el caso del ejercicio del calendario". En general, los expertos presentaron un alto nivel de satisfacción con la guía de trabajo para la familia, solo uno de ellos realizó un comentario de mejora, con respecto al ejercicio de usar los platos para trabajar las fracciones, indicando, "Se debe especificar que todos los platos deben ser del mismo tamaño". Los comentarios de los expertos se consideraron en el diseño final de la guía.

En lo que sigue se presenta el análisis y resultados de la evaluación de la guía de apoyo familiar, que realizaron los apoderados del Tercer Año Básico, además cuatro profesores del colegio y el encargado de la Unidad Técnico Pedagógica del establecimiento.

\section{ANÁLISIS Y RESULTADOS DE LA EVALUACIÓN DE LA GUÍA DE APOYO}

El análisis de los resultados de algunos aspectos generales de la guía, muestra el siguiente panorama del curso. La mayor parte del rango etario de las familias está entre los 30 y 40 años; es decir, la gran mayoría de los apoderados están categorizados como adultos jóvenes según clasificación del Instituto Nacional de Estadística.

En cuanto a la participación desde una visión de género, se observa una tendencia casi mayoritaria del sexo femenino (81\%), siguiendo con la visión de sociedad tradicional, en que las mujeres se encargaban mayoritariamente de la educación de sus hijos (Flamey, Gubbins y Morales, 1999). Esto se ve claramente respaldado con los resultados de la pregunta ¿Qué parentesco tiene con el estudiante?, en el cual la mayoría de las personas que respondió la encuesta de satisfacción, manifiesta tener una relación materna con el estudiante (73\%).

A continuación se presentan los resultados de la guía de satisfacción, según aspectos generales, contenidos matemáticos y relación familia-escuela.

\subsection{ASPECTOS GENERALES DE LA GUÍA}

Los resultados obtenidos de la pregunta, ¿El formato de la guía le resultó fácil de entender?, entrega una tendencia clara de alto nivel de satisfacción de los padres y apoderados, en que el 100\% manifestaron estar "de acuerdo" o "muy de acuerdo" con el formato que presentaba la guía de apoyo a las familias. En cuanto al lenguaje usado en la guía fue fácil de comprender, el $98 \%$ de los encuestados entregó un alto nivel de satisfacción y el 2\% restante manifestó un nivel de percepción neutra o indecisa a través del indicador "ni de acuerdo, ni en desacuerdo"; como se ilustra en la Figura 1. 


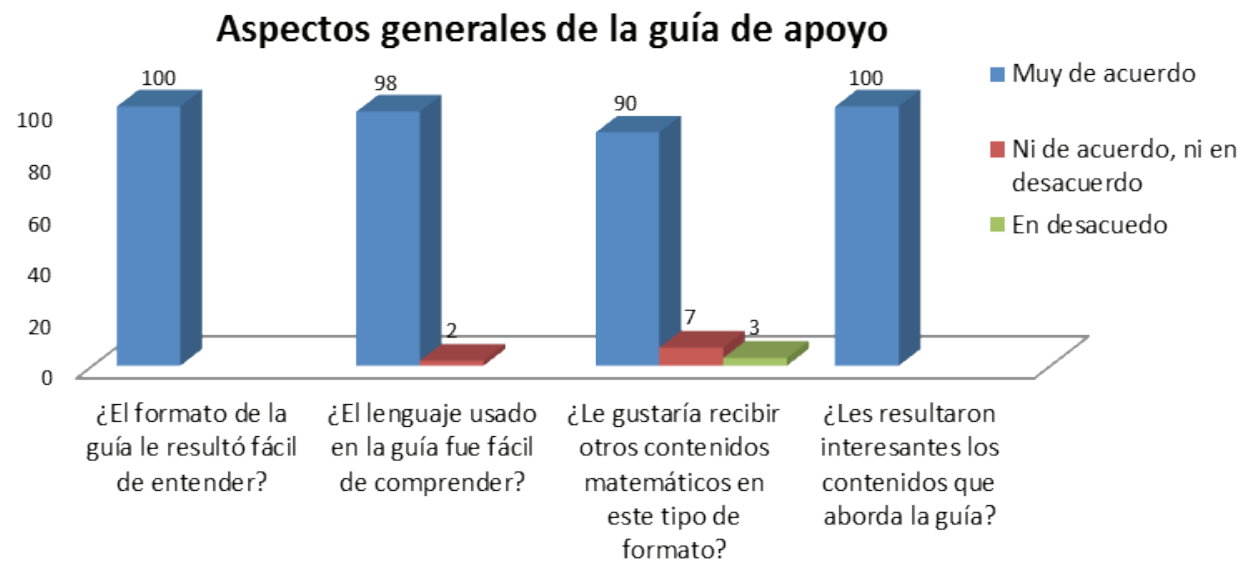

Figura 1. Apreciaciones generales de la guía de apoyo

Los apoderados indicaron en un $90 \%$ que les gustaría recibir otros contenidos matemáticos en el formato indicado, el $7 \%$ señalo un nivel de satisfacción neutra o indiferente y el 3\% de los apoderados señaló un nivel de satisfacción bajo; es decir, no le gustaría recibir otros contenidos matemáticos en este formato. Respecto al interés de los contenidos que aborda la guía, el 100\% de ellos señaló estar "de acuerdo "o "muy de acuerdo" con los contenidos presentes en la guía. En resumen, se puede establecer que en cuanto al formato de la guía de apoyo a las familias (en relación al contenido de fracciones), para el Tercer Año Básico, el grado de satisfacción de los padres o apoderados fue mayoritariamente alto.

\subsection{CONTENIDO MATEMÁTICO}

Respecto a la pregunta si los ejercicios presentes en la guía fueron adecuados a los temas que los alumnos trabajan en el colegio, el grado de satisfacción de los apoderados fue alto, llegando al 98\% los indicadores "de acuerdo" o "muy de acuerdo", respectivamente. En cuanto al 2\% restante manifestó un grado de satisfacción neutral o indiferente, seleccionando el indicador "ni de acuerdo, ni en desacuerdo".

Sobre si las actividades de fracciones fueron fáciles, hubo una clara inclinación hacia un grado de satisfacción positiva (99\%), sólo $1 \%$ indicó estar "en desacuerdo". Respecto a la claridad para entender las actividades planteadas se presentan resultados similares a las preguntas anteriores, en que el grado de satisfacción de los padres y apoderados es alto (98\%), y un $2 \%$ señala un grado de satisfacción neutro o indiferente, a través del indicador "ni de acuerdo, ni en desacuerdo".

Sobre las direcciones Web sugeridas los apoderados mostraron una tendencia más dispersa, en el cual el $65 \%$ señaló un alto grado de satisfacción seleccionando los indicadores "muy de acuerdo" o "de acuerdo" respectivamente, el 30\% manifestó un grado de satisfacción neutro o indiferente y el 5\% seleccionó el indicador "en desacuerdo". 
A modo de conclusión en este ítem, los padres y apoderados mantuvieron la tendencia del ítem anterior, inclinando sus respuestas hacia un grado alto de satisfacción. No obstante, en la pregunta sobre las páginas de Internet sugeridas, el porcentaje de respuestas neutras o indiferentes fue del $30 \%$, lo cual puede darnos algunas luces de que los padres y apoderados no se dieron el tiempo para revisar dichas páginas o definitivamente no les parecieron interesantes, debido a que el contenido ya había sido estudiado por parte de los estudiantes. En la Figura 2 se reportan los resultados mencionados.

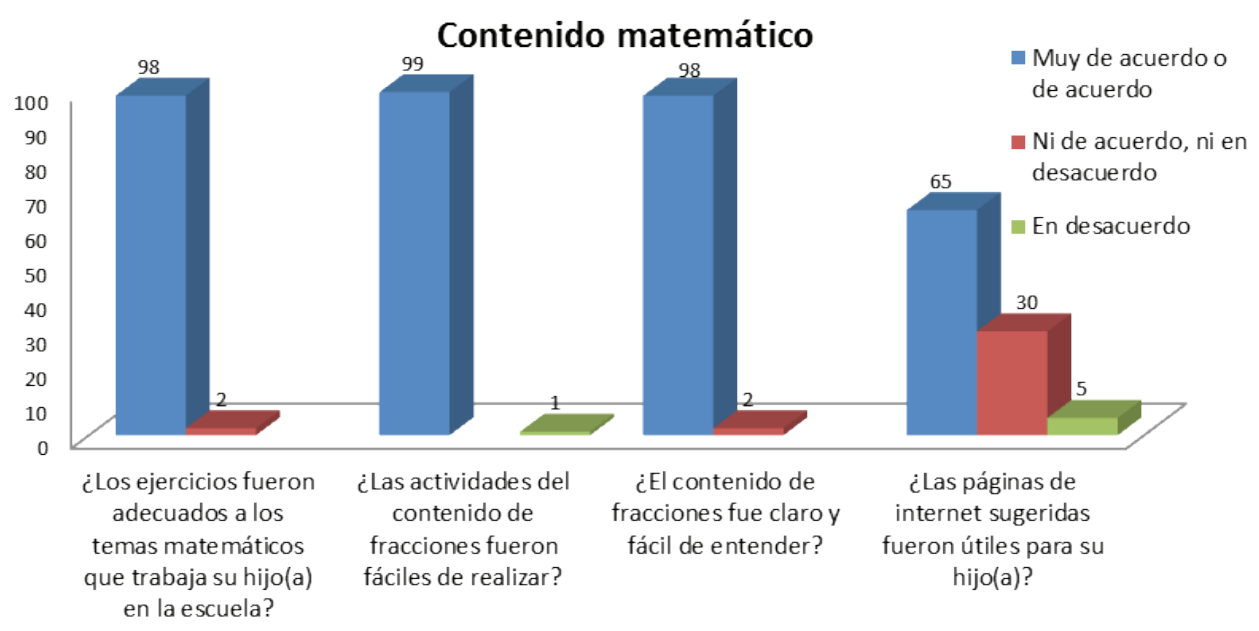

Figura 2. Apreciaciones generales sobre el contenido matemático

\subsection{RELACIÓN FAMILIA-ESCUELA}

El objetivo de incluir este ítem dentro de la encuesta de satisfacción era tener una visión de las percepciones que tenían los padres y apoderados sobre la relación de las familias con el colegio en que asisten sus hijos. Con respecto a qué si el apoderado cree que una forma de mejorar la educación es construyendo una buena relación entre familia- escuela, ellos manifestaron un alto nivel de satisfacción, en el cual el 100\% de las respuestas obtenidas estuvieron distribuidas entre los indicadores "de acuerdo" o "muy de acuerdo". Además sostienen en un 95\% la importancia de participación como apoderados en la escuela, sólo un 5\% manifestó un grado de satisfacción neutra o indiferente, seleccionando el indicador "ni de acuerdo, ni en desacuerdo".

Con respecto a la pregunta, ¿Si la escuela fuera más inclusiva con las familias, usted se sentiría más motivado de participar en el proceso de enseñanza aprendizaje de su hijo(a)? los padres y apoderados presentaron una tendencia clara hacia un alto grado de satisfacción, siendo un $97 \%$ de los encuestados los que seleccionaron el indicador "muy de acuerdo" y el 3\% restante seleccionó el indicador "ni de acuerdo, ni en desacuerdo" evidenciando con ello su grado de satisfacción neutra o indiferente.

A la pregunta, ¿Es la excesiva carga laboral uno de los principales motivos de la baja participación de las familias en los centro educativos? Los padres y apoderados mostraron 
un bajo nivel de satisfacción con respecto a esta aseveración, siendo solo un $47 \%$ de los encuestados los que seleccionaron los indicadores "en desacuerdo", un 35\% manifestó un grado de satisfacción neutra o indiferente y un $18 \%$ seleccionó el indicador "muy de acuerdo". En la Figura 3 se sintetizan los resultados obtenido sobre la relación familia y escuela.

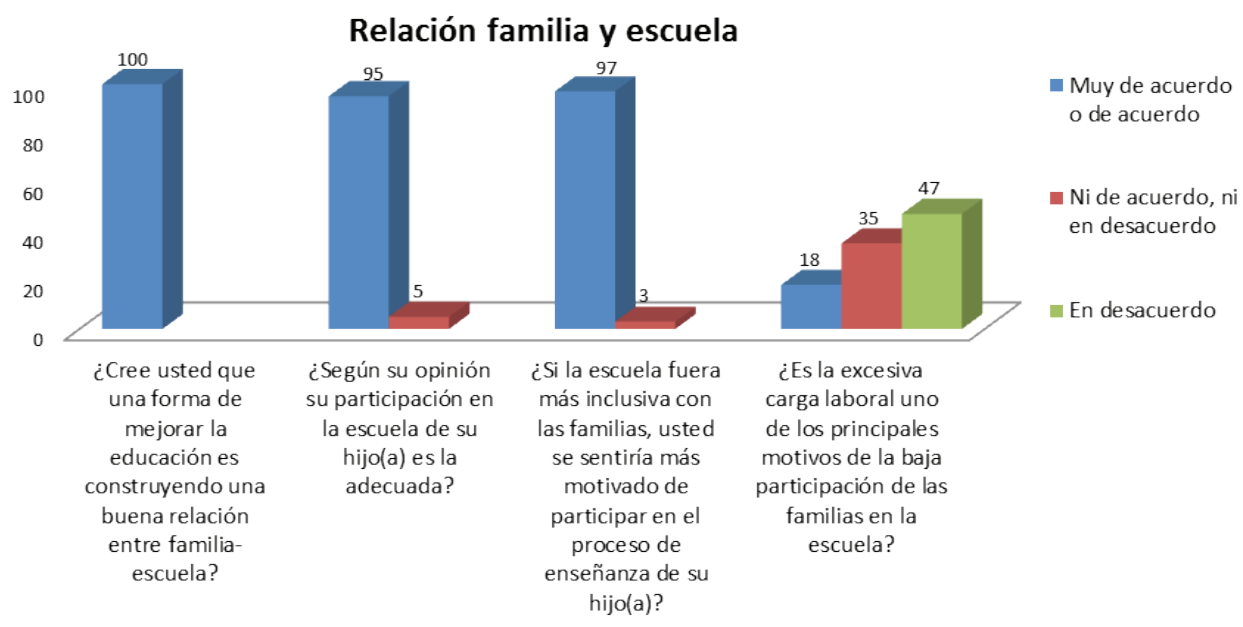

Figura 3. Apreciaciones sobre la relación familia y escuela

\subsection{SUGERENCIAS GENERALES}

Los apoderados indicaron sobre la guía de apoyo que les gustaría recibir este tipo de material en otros contenidos matemáticos y/o asignaturas (especialmente lenguaje e historia). Indicando que en matemática se pueden tratar contenidos complejos en la enseñanza como lo son las multiplicaciones y divisiones.

Sobre la claridad matemática del material, los apoderados indicaron que "mi hijo quedó muy claro con el contenido de fracciones", "la encontré divertida y práctica", "las actividades fueron fáciles y entretenidas"; se aprecia que las actividades de la guía fueron de fácil comprensión para las familias y sencillas de ejecutar.

Las actividades que los apoderados recomiendan agregar al material de apoyo son ejemplos o más ejercicios. Respecto si les quitarían algo se indica que "no le agregaría nada, está de acuerdo a la enseñanza de mi hijo". En general, la mayoría de las familias manifestaron una percepción positiva hacia la guía de apoyo.

\subsection{ANÁLISIS DE LAS PERCEPCIONES DE LOS DOCENTES Y DEL ENCARGADO DE LA UNIDAD TÉCNICO PEDAGÓGICA}

La guía de apoyo también fue evaluada por cuatro docentes y un encargado de la Unidad Técnico Pedagógica del colegio. Sobre los aspectos formales de la guía, la totalidad de 
evaluadores considera que el lenguaje es adecuado a los padres y apoderados. Asimismo, todos indican que la guía puede ser aplicada a familias de cualquier grupo socioeconómico; ya que, las actividades están contextualizadas a situaciones habituales de los hogares.

El diseño de la guía, fue evaluado con un alto nivel de satisfacción (4 de 5 personas), destacándose que las ilustraciones, como llamativas y motivadora para los estudiantes y para a las familias. Además se acentúa que la guía no sea extensa; ya que, cuando los textos son muy largos las familias no los usan (manifestó el en cargado de UTP del colegio).

Con respecto a si el contenido de la guía es correspondiente con los objetivos de aprendizajes de $3^{\circ}$ Año Básico, 4 de 5 personas indicaron estar de acuerdo con la relación, el otro académico no recordaba los objetivos de aprendizajes para el nivel escolar por lo cual no contestó a la pregunta. De la aseveración, las instrucciones de la guía son claras y fáciles de comprender 4 de cada 5 académicos indican estar de acuerdo.

Sobre los aspectos matemáticos los 5 académicos indican que la guía es adecuada para trabajar con las familias, indicando que las fracciones es un tema que siempre los estudiantes tienen dificultad para aprender. Respecto a si los contenidos se presentan de forma clara, 4 de 5 evaluadores están de acuerdo y el otro docente expresa que hay problemas en algunos enunciados que pueden ser confusos a la hora de abordarlos.

Sobre las páginas de internet presentadas en la guía, 3 de 5 evaluadores indican que son adecuadas para los padres y apoderados. Los restantes académicos mostraron un nivel de satisfacción neutro o indiferente, ya que no revisaron de forma exhaustiva las páginas de Internet sugeridas para profundizar en el contenido de las fracciones.

\subsubsection{Respecto a la relación de familia y escuela}

Los cinco evaluadores destacan que la guía de apoyo constituye una adecuada estrategia para vincular a la familia en el proceso de enseñanza aprendizaje. Además, 4 de 5 profesores indican que es una adecuada estrategia para generar vínculos entre la familia y el profesor de la asignatura. El otro evaluador indica que cualquier estrategia que se utilice para afianzar lazos con las familias se agradece, ya que ellos están conscientes que es un punto que no es abordado en el colegio.

En cuanto a la factibilidad de implementar este tipo de guías por lo menos una vez al mes y a todas las asignaturas, los docentes manifestaron un bajo nivel de satisfacción (4 de 5). Según las opiniones manifestadas la excesiva carga laboral es el impedimento principal, no les sería factible poder hacer la construcción de este tipo de guía de forma mensual, mucho menos poder aplicarlas a todas las asignaturas y cursos que ellos tienen.

A modo de conclusión, y luego de analizar la información recogida en la encuesta de validación de contenidos, podemos inferir que existe un alto nivel de satisfacción de los docentes y UTP consultados, lo cual nos da claros indicios que ellos valoran y están conscientes que es de suma importancia incluir a las familias en el proceso de enseñanza aprendizaje de sus hijos.

Otro indicador que se destaca de este análisis, aunque no tiene una valoración positiva, es que los profesores y encargados e UTP no están dispuestos a dedicar tiempo para construir guías de apoyo, ya sea en el área de matemáticas u otras asignaturas. Esto se debe a la alta carga laboral que manifiestan tener. 


\section{CONCLUSIONES}

A partir del análisis de las diversas propuestas para trabajar con las familias en las escuelas, realizadas por instituciones nacional como el MINEDUC e instituciones internacionales como UNICEF, se puede advertir que a pesar de que existe una cierta intención de impulsar y fortalecer el trabajo con las familias de los estudiantes que forman parte de los centros educativos, las propuestas existentes presentan una estructura de trabajo más bien general y poco contextualizadas, siento las mismas propuestas las que se utilizan en la diversidad de establecimientos del país.

Bajo este contexto se decidió llevar a cabo una propuesta metodológica de apoyo a las familias, para abordar las debilidades que presentaban estudiantes de Tercer Año Básico de un colegio regular chileno.

A partir de los antecedentes recogidos en la etapa de diagnóstico y bajo la orientación de las Bases Curriculares y Programas de Estudio para Tercero Básico en la asignatura de matemática, se realizó la confección de dicha guía. Los resultados obtenidos muestran que la guía de apoyo familiar produjo un alto nivel de satisfacción, no solo de las familias que constituyen el Tercer Año Básico, sino que también de profesores y el encargado de Unidad Técnica Pedagógica del colegio.

Las familias de los estudiantes manifestaron un alto grado de satisfacción con respecto al tipo de ejercicios que contenía la guía de apoyo, utilizando para calificar conceptos como: "divertida, lúdica y entretenida". A su vez solicitaron poder utilizar este tipo de estrategias de trabajo en otros contenidos matemáticos como, multiplicaciones y divisiones, e incluso solicitaron la aplicabilidad de este formato de guía de apoyo en otras asignaturas.

La buena disposición y compromiso de las familias llegó a tal punto, que muchas de ellas enviaron la guía resuelta de regreso al colegio con el estudiante, adjuntando el material didáctico propuesto en la guía (memorice de fracciones). En este trabajo no reportamos resultados de la implementación del material.

En este último tiempo, diversas instituciones como el MINEDUC, UNICEF y la Agencia de Calidad de la Educación, entre otras, han puesto su mirada en valorar y reforzar la alianza de las escuelas con las familias. Por ejemplo, la Agencia de Calidad de la Educación (2015) presenta una guía con orientaciones para mejorar los aprendizajes integrales, en dicha guía se encuentra un capítulo completo que tiene por objetivo dar una serie de orientación de cómo y cuándo integrar a los padres y apoderados en el proceso de enseñanza aprendizaje de sus hijos.

Si las instituciones que actualmente apoyan y/o regulan la educación del país, están haciendo un esfuerzo por volver a integrar a las familias en los centros educativos, ¿Dónde está la falencia? ¿En qué parte del eslabón se pierden las ideas o ganas de integrar a las familias? Luego de analizar los resultados de la encuesta de satisfacción aplicada a los profesores y jefe de UTP del colegio, quedó evidenciado un punto clave sobre la postura de los profesores en cuanto a medidas para acercar o fortalecer la relación con las familias. Cuando se consultó sobre la guía de apoyo a las familias, la totalidad de los docentes manifestó un alto grado de satisfacción con ella, pero cuando se les consultó por la factibilidad de poder diseñar y aplicar esta guía en todas las asignaturas y de forma periódica durante el año escolar, ellos indican que no es factible por la excesiva carga laboral que manifiestan tener.

Desde diversas disciplinas se dan resultados irrefutables sobre lo beneficioso que es para cada estudiante sentir el respaldo familiar y de la escuela durante su extenso proceso 
de aprendizaje. Los profesores son los principales interlocutores de la escuela con las familias de los estudiantes, cuya función principal actualmente es solo informativa. Bajo este contexto las familias pierden la estimulación de participar en los centros educativos y están muy lejos de sentirse una pieza fundamental para lograr una educación de calidad.

Según los antecedentes expuestos en esta investigación, hay dos posibles caminos a seguir para lograr una verdadera inclusión de las familias en el proceso de enseñanza aprendizaje, por un lado está la opción de no hacer nada y seguir con el discurso de muchos docentes, directivos y familias, ¡No tengo tiempo! ¡Mi carga laboral es excesiva! En el extremo opuesto esta la opción de convertirnos en verdaderos agentes de cambios, sin excusa alguna, posicionarnos como agentes activos en este importante proceso educativo de cada estudiante, con el objetivo que el colegio no solo se convierta en una escuela democrática, en que cada agente que la compone sea escuchado e integrado en ella, sino que cada colegio se trasforme en un centro educativo cohesionado y conectado con cada agente involucrado en el proceso de enseñanza aprendizaje.

\section{REFERENCIAS BIBLIOGRÁFICAS}

Agencia de Calidad de la Educación. (2015). Resultados Educativos. Santiago de Chile: Gobierno de Chile.

Arancibia, V., Herrera, P. y Strasser, K. (1999). Manual de psicología educacional. Santiago: Pontificia Universidad Católica de Chile.

Bolívar, A. (2006). Familia y escuela: dos mundos llamados a trabajar en común. Revista de Educación, 339, 191-146.

Burrows, F. (2006). Familia y proceso de aprendizaje. Estudio "Prácticas sociales a nivel familiar que tienen relación con los aprendizajes de niños y niñas del nivel preescolar y del primer ciclo escolar básico, de Villarrica y Pucón”. Santiago de Chile: Pontificia Universidad Católica de Chile.

Cohen, L., Manion, L. \& Morrison, K. (2011). Research methods in education. Londres: Routledge.

Coleman, J. y Hendry. L. (2003). Psicología de la adolescencia. (4ºdición). Madrid: Morata.

Corthorn, C. y Pérez, L. (2005). Educación de Calidad para nuestros hijos: Guía de apoyo para la familia. Santiago de Chile: MINEDUC - UNICEF.

Decreto Nº12/2005 del Código del Derecho Canónico. Documento sin publicar.

Flamey, G., Gubbins, V. y Morales, F. (1999). Los Centros de Padres y Apoderados: Nuevos Actores en el Control de la Gestión Escolar. Santiago de Chile: CIDE.

González, J. (2010). Recursos y materiales didácticos manipulativos para fracciones y números decimales. Matemática y su didáctica. Málaga: Universidad de Málaga.

González- Pienda, J. y Núñez, L. (2005). La incidencia de los padres y la incidencia en el rendimiento de sus hijos. Revista de Psicología y Educación, vol.1, 115-134.

Grant, B. \& Ray, J. (2013). School and community collaboration. London: England.

Gubbins, V. (2016). Relación Familias y Escuelas: ¿Por qué y para qué? Santiago de Chile: Universidad Finis Terrae.

Gurdían, A. (2007). El Paradigma Cualitativo en la Investigación Socio-Educativa. Costa Rica: Colección Investigación y Desarrollo.

Heuvel-Panhuizen, M. (2002). Realistic mathematics education as work in progress. In Fou-Lai Lin (Eds.). Common sense in mathematics education. Proceedings of 2001. The Netherlands and Taiwan Conference on Mathematics Education (pp. 1- 43). Taiwan: National Taiwan Normal University. 
Kaukab, S. (2016). The impact of parent/family involvement on student' learning outcomes. International Journal of Research - Granthaalayah, vol.4 (10), 72-81.

Kotliarenco, M. A. y Cortés, M. (2001). Importancia del rol de los padres como principales educadores de sus hijos e hijas. Santiago de Chile: UNESCO.

De León. B. (2011). La relación familia-escuela y su repercusión en la autonomía y responsabilidad de los niñas/as. XII Congreso Internacional de Teoría de la Educación (pp.1-20). Barcelona, España. Recuperado el 5 de abril de 2012 https://extension.uned.es/archivos_publicos/webex_ actividades/5385/repercusiones8.pdf

Lucia, V. \& Breslau. N. (2006). Family cohesion and children's behavior problems: A longitudinal investigation. Psychiatry Research, vol.14, 141 - 149.

doi:10.1016/j.psychres.2005.06.009

Ministerio de Educación (2016). Cuenta conmigo $3^{\circ}$ y $4^{\circ}$ Básico. Santiago de Chile: Ministerio de Educación.

Ministerio de Educación (2013a). Aprendiendo en familia. Guía para apoyar a los padres y madres en la educación de sus hijos e hijas. Santiago de Chile: Gobierno de Chile.

Ministerio de Educación (2013b). Bases Curriculares. Santiago de Chile: Gobierno de Chile.

Ministerio de Educación (2011). Cartilla para padres, madres y apoderados/as. Santiago de Chile: Gobierno de Chile.

Obiol, S. (2012). El cambio familiar y el proceso educativo. Madrid: McGrawHill.

Padua, J. (2000). Técnicas de investigación aplicadas a las ciencias sociales. México: Fondo de cultura económica.

PISA (2012). Results: What Students Know and Can Do Student-Performance in Mathematics. Reading and Science (Vol.1). PISA: OECD Publishing. doi: 10.1787/9789264201118-en

Raczynski, D. y Muñoz, G. (2005). Efectividad escolar y cambio educativo. En condiciones de pobreza en Chile. Santiago de Chile: Gobierno de Chile.

Razeto, A. (2016). Estrategias para promover la participación de los padres en la educación de sus hijos: el potencial de la visita domiciliaria. Estudios Pedagógicos, vol.42 (2), 449-462. Recuperado el 10 de mayo de 2017 desde http://mingaonline.uach.cl/scielo.php?script=sci_ arttext\&pid=S0718-07052016000200026\&lng=es\&nrm=iso

Rodrigo M. y Palacios J. (2005). Familia y Desarrollo Humano. Madrid: Alianza.

Romagnoli, C. y Cortese, I. (2015). ¿Cómo la familia influye en el aprendizaje y rendimiento escolar? Santiago de Chile: Valoras UC. Recuperado el 10 de marzo de 2017 desde www.valoras.uc.cl

UNESCO (2004). Participación de las familias en la educación infantil latinoamericana. Santiago de Chile: UNICEF.

UNICEF (2005). Guía de apoyo para la familia. Santiago de Chile: UNICEF.

UNICEF/MINEDUC (2009). Reuniones de apoderados. Acercando a las familias la escuela. Santiago de Chile: Gobierno de Chile.

UNICEF/MINEDUC (2007). Manual para profesores Jefe. Santiago de Chile: UNICEF.

Valdés, X. y Valdés, T. (Eds.). (2005). Familia y vida privada ¿transformaciones, tensiones, resistencias o nuevos sentidos? Santiago: CEDEM/FLACSO.

Villarroel, G. y Sánchez, X. (2002). Relación familia y escuela: un estudio comparativo en la ruralidad. Estudios Pedagógicos, vol.28, 123-141. Recuperado el 15 de enero de 2017 desde http://www.scielo.cl/scielo.php?script=sci_arttext\&pid=S0718-07052002000100007 
